\title{
MODELAGEM DE MÁQUINA PNEUMÁTICA RECOLHEDORA DE FRUTOS DE CAFÉ EM TERREIRO USANDO ANÁLISE POR ELEMENTOS FINITOS
}

\author{
ANDERSON C. MAGALHÃES ${ }^{1}$, MAURI M. TEIXEIRA ${ }^{2}$, SANDRA M. COUTO ${ }^{3}$, \\ RICARDO C. DE RESENDE ${ }^{4}$
}

\begin{abstract}
RESUMO: Teve-se o objetivo de desenvolver um modelo matemático por meio de análise de elementos finitos, utilizando o programa computacional ANSYS ${ }^{\circledR}$, versão 5.7, para otimizar o projeto de máquina recolhedora de frutos de café no terreiro. A modelagem da máquina foi realizada com base no levantamento das características aerodinâmicas dos frutos de café e da vazão de ar necessária para o transporte pneumático dos frutos. Foram obtidas, experimentalmente, as pressões estáticas nos dutos da máquina, sendo esses valores comparados com os resultados determinados pelo programa ANSYS, no intuito de validar o modelo. Com base nos resultados numéricos obtidos, concluiu-se que a modelagem desenvolvida apresentou resultados próximos aos determinados experimentalmente, obtendo erro relativo médio nos valores simulados de pressão de $9,2 \%$. Por meio da modelagem, identificaram-se faixas de pressão que dificultariam o transporte pneumático dos frutos de café em alguns pontos da máquina. Esses problemas foram corrigidos e, com isso, o fluxo de ar proporcionado pelo ventilador foi suficiente para succionar os frutos de café no terreiro e transportá-los para dentro do reservatório da máquina. A modelagem desenvolvida atendeu às necessidades propostas no trabalho para o recolhimento dos frutos de café utilizando transporte pneumático eficientemente.
\end{abstract}

PALAVRAS-CHAVE: máquina agrícola, simulação, projeto.

\section{MODELING OF PNEUMATIC MACHINE COLLECTOR OF COFFEE FRUITS ON YARD USING FINITE ELEMENTS ANALYSIS}

\begin{abstract}
The objective of this work was the development of a mathematical model by finite element analysis, using the software ANSYS $^{\circledR}$, version 5.7, to optimize the design of a machine which collects coffee fruits on a yard. The machine modeling was based on the aerodynamic characteristics of coffee fruits and on the air flow necessary to pneumatically transport the coffee fruits. The static pressures on the machine ducts was obtained experimentally and these values were compared with the results obtained by the software ANSYS to validate the modeling. Based on the results, it was concluded that the modeling using the software ANSYS had similar results to the ones obtained experimentally, with a relative mean error of $9.2 \%$ for the simulated pressure values. Through the modeling, it was identified pressure ranges that would make the coffee fruits pneumatic transport more difficult, at some parts inside the machine. These problems were corrected and the air flow supplied by the fan was sufficient to collect coffee fruits on the ground by suction. The developed modeling fulfilled the requirements of this work which was to collect coffee fruits on the yard using pneumatic transport efficiently.
\end{abstract}

KEYWORDS: collecting machine, simulation, design.

\footnotetext{
${ }^{1}$ Eng ${ }^{o}$ Agrícola, Prof. Assistente Doutor, Faculdade de Zootecnia, Câmpus de Dracena, UNESP, Fone: (0XX18) 3821.8200, Ramal 8221, Fax: (0XX18) 3821.8208, amchagas@ dracena.unesp.br

${ }^{2}$ Engo Agrônomo, Prof. Dr., Departamento de Engenharia Agrícola, UFV, Viçosa - MG.

${ }^{3}$ Bacharel em Matemática, Profa. Ph.D., Departamento de Engenharia Agrícola, UFV, Viçosa - MG.

${ }^{4}$ Engo Mecânico, Prof. Ph.D., Departamento de Engenharia Agrícola, UFV, Viçosa - MG.

Recebido pelo Conselho Editorial em: 27-7-2005
}

Aprovado pelo Conselho Editorial em: 17-5-2006 


\section{INTRODUÇÃO}

No estudo de máquinas agrícolas, o conhecimento das propriedades e das características físicas dos grãos é essencial para o seu desenvolvimento e para a sua construção. Esse fato é importante, porque propicia operações mais eficientes, tais como: limpeza, transporte, secagem, beneficiamento e armazenagem dos produtos agrícolas (SILVA, 1995; SRIVASTAVA et al., 1996).

Além da determinação das características físicas dos produtos, a modelagem matemática pode ser uma ferramenta necessária no desenvolvimento de uma máquina agrícola. $\mathrm{O}$ aumento da velocidade, da capacidade de processamento dos computadores e da necessidade de estudos de máquinas sofisticadas são os principais fatores que contribuíram para o desenvolvimento dessas técnicas de análise e projeto de máquinas (NORTON, 1992; KNIGHT, 1993).

A análise por elementos finitos consiste na divisão de um corpo, utilizando malha de elementos e funções aproximadas para descrever o comportamento das variáveis envolvidas no problema (ZHU et al., 1989; REDDY, 1993). Conforme SEGERLIND (1984) e HUEBNER et al. (1995), essa técnica é utilizada tanto para análise de tensões, como para problemas envolvendo transferência de calor, mecânica dos fluidos e outras aplicações.

O uso de computadores tem proporcionado o desenvolvimento de "protótipos virtuais", em que determinada máquina ou sistema é modelado mediante um programa computacional; primeiramente o seu comportamento é simulado sob diferentes condições de funcionamento, antes que ela entre em linha de produção. Dessa forma, não somente são reduzidos os custos, mas também o tempo necessário para projetar máquinas, o número de protótipos a serem construídos e os testes experimentais a serem realizados. Diante disso, máquinas tecnicamente mais eficientes e de menor custo poderão ser desenvolvidas e o seu projeto ser otimizado (HAGHIGHI et al., 1990; GARCIA, 1998).

Além disso, as máquinas agrícolas podem facilitar o processo de transporte de grãos no terreiro e contribuir para a obtenção de um produto final de melhor qualidade. $O$ sistema de transporte pneumático, desde que devidamente projetado, pode ser, por exemplo, uma importante opção para o transporte de frutos do café no processo de secagem, sem causar danos consideráveis ao produto.

Considerando a importância da modelagem, este trabalho teve como objetivo desenvolver um modelo, usando elementos finitos, para determinar a dinâmica dos fluxos de ar no transporte pneumático de máquina recolhedora de café.

\section{MATERIAL E MÉTODOS}

A máquina recolhedora foi modelada nos Laboratórios de Armazenamento e de Mecanização Agrícola do Departamento de Engenharia Agrícola (DEA) da Universidade Federal de Viçosa.

O desenvolvimento envolveu o levantamento das características aerodinâmicas dos frutos de café e da vazão de ar necessária para o transporte pneumático. Os valores calculados da velocidade terminal dos frutos de café por MAGALHÃES (2003), variaram de 15,5 a $13,5 \mathrm{~m} \mathrm{~s}^{-1}$ para a faixa de teor de água de 41,1 a $28,7 \%$ (b.u.). Para a modelagem, essa faixa constitui o ponto crítico, devido ao elevado teor de água nos frutos de café que aumenta a velocidade terminal dos frutos, quando comparado aos frutos de café seco para o recolhimento (teor de água entre 11 e $13 \%$ b.u.). A partir dessas informações, foi utilizado o programa ANSYS, versão 5.7, para a simulação do escoamento na máquina recolhedora. $\mathrm{O}$ projeto dessa máquina foi elaborado de acordo com o fluxograma de recolhimento dos frutos de café (Figura 1).

Para determinar o seu comportamento dinâmico, foram utilizadas as dimensões reais do projeto da máquina recolhedora de frutos de café (Figura 2). 


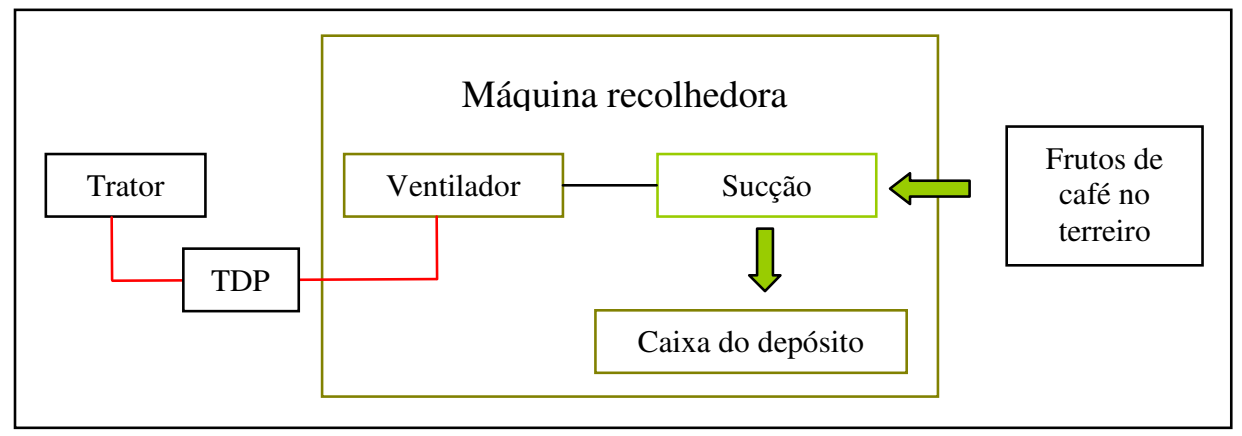

FIGURA 1. Fluxograma de execução do projeto.

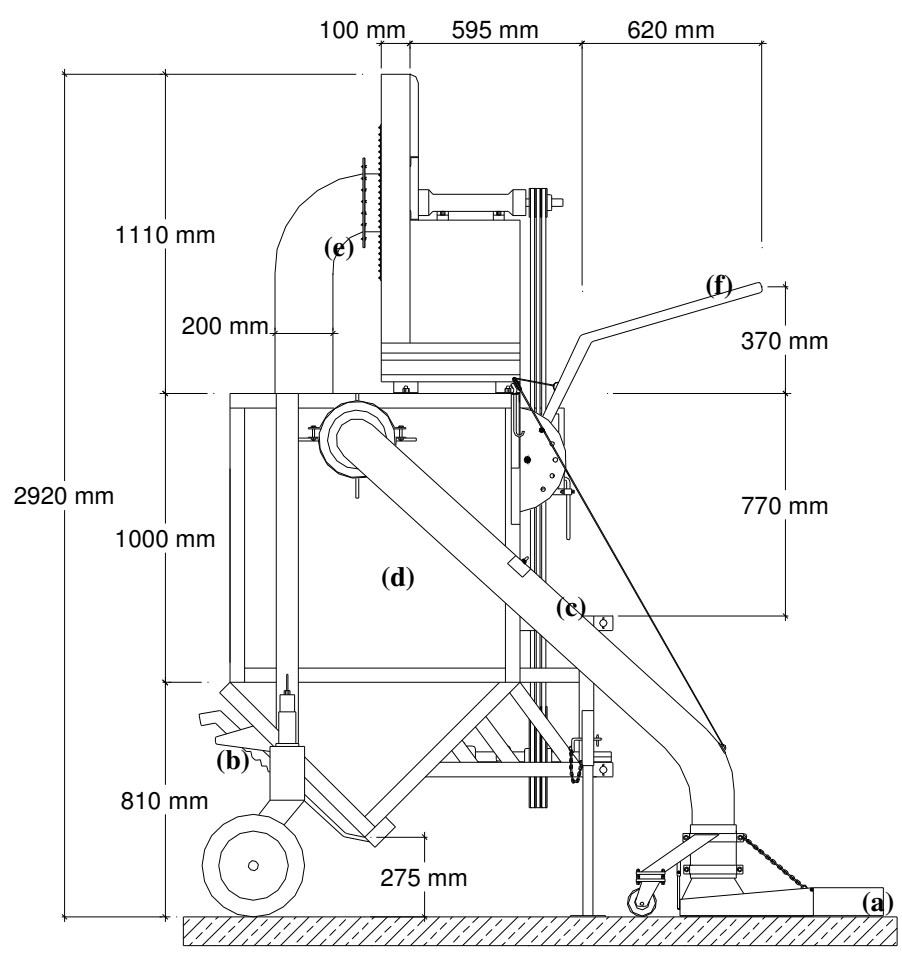

FIGURA 2. Vista lateral direita da máquina recolhedora de frutos de café. Componentes: (a) caixa captadora; (b) alavanca que regula o fluxo de descarga do produto; (c) duto de entrada do produto; (d) caixa do depósito; (e) duto de entrada do fluxo de ar no ventilador, e (f) alavanca que ergue o duto.

\section{Desenvolvimento do modelo matemático da máquina recolhedora de café}

Para a simulação computacional da máquina, foi utilizado modelo matemático baseado nas equações de Navier-Stokes (equações de quantidade de movimento mais equação de conservação da massa) e o Software ANSYS ${ }^{\circledR} 5.7$ para obtenção dos resultados numéricos. Esse software soluciona os problemas de engenharia, pelo método dos elementos finitos, com diversos recursos de geração de malha e definição de modelo na sua parte de pré-processamento (ANSYS, 2001).

O escoamento de ar por meio da máquina é considerado incompressível e pode ser modelado matematicamente pelas equações:

- Conservação da massa:

$\frac{\partial \rho}{\partial t}+\frac{\partial\left(\rho v_{x}\right)}{\partial x}+\frac{\partial\left(\rho v_{y}\right)}{\partial y}+\frac{\partial\left(\rho v_{z}\right)}{\partial z}=0$

- Quantidade de movimento: 


$$
\begin{array}{r}
\frac{\partial\left(\rho v_{x}\right)}{\partial t}+\frac{\partial\left(\rho v_{x} v_{x}\right)}{\partial x}+\frac{\partial\left(\rho v_{y} v_{x}\right)}{\partial y}+\frac{\partial\left(\rho v_{z} v_{x}\right)}{\partial z}=\rho g_{x}-\frac{\partial p}{\partial x}+R_{x}+\frac{\partial}{\partial x}\left(\mu_{e} \frac{\partial v_{x}}{\partial x}\right)+ \\
+\frac{\partial}{\partial y}\left(\mu_{e} \frac{\partial v_{x}}{\partial y}\right)+\frac{\partial}{\partial z}\left(\mu_{e} \frac{\partial v_{x}}{\partial z}\right)+T_{x} \\
\frac{\partial\left(\rho v_{y}\right)}{\partial t}+\frac{\partial\left(\rho v_{x} v_{y}\right)}{\partial x}+\frac{\partial\left(\rho v_{y} v_{y}\right)}{\partial y}+\frac{\partial\left(\rho v_{z} v_{y}\right)}{\partial z}=\rho g_{y}-\frac{\partial p}{\partial y}+R_{y}+\frac{\partial}{\partial x}\left(\mu_{e} \frac{\partial v_{y}}{\partial x}\right)+ \\
+\frac{\partial}{\partial y}\left(\mu_{e} \frac{\partial v_{y}}{\partial y}\right)+\frac{\partial}{\partial z}\left(\mu_{e} \frac{\partial v_{y}}{\partial z}\right)+T_{y} \\
\frac{\partial\left(\rho v_{z}\right)}{\partial t}+\frac{\partial\left(\rho v_{x} v_{z}\right)}{\partial x}+\frac{\partial\left(\rho v_{y} v_{z}\right)}{\partial y}+\frac{\partial\left(\rho v_{z} v_{z}\right)}{\partial z}=\rho g_{z}-\frac{\partial p}{\partial z}+R_{z}+\frac{\partial}{\partial x}\left(\mu_{e} \frac{\partial v_{z}}{\partial x}\right)+ \\
+\frac{\partial}{\partial y}\left(\mu_{e} \frac{\partial v_{z}}{\partial y}\right)+\frac{\partial}{\partial z}\left(\mu_{e} \frac{\partial v_{z}}{\partial z}\right)+T_{z}
\end{array}
$$

em que,

$\mathrm{v}_{\mathrm{x}}, \mathrm{v}_{\mathrm{y}} e \mathrm{v}_{\mathrm{z}}$ - componentes do vetor velocidade nas direções $\mathrm{x}, \mathrm{y}$ e $\mathrm{z}$, respectivamente;

$\rho$ - massa específica do ar;

$\mathrm{x}, \mathrm{y}$ e $\mathrm{z}$-coordenadas cartesianas;

T - tempo;

P - pressão;

$\mathrm{g}_{\mathrm{x}}, \mathrm{g}_{\mathrm{y}}$ e $\mathrm{g}_{\mathrm{z}}$ - componentes da aceleração da gravidade nas direções $\mathrm{x}, \mathrm{y}$ e $\mathrm{z}$, respectivamente;

$\mu_{\mathrm{e}}-$ viscosidade efetiva, e

$\mathrm{T}_{\mathrm{x}}, \mathrm{T}_{\mathrm{y}}$ e $\mathrm{T}_{\mathrm{z}}$ - termos de perda viscosa, nas direções $\mathrm{x}, \mathrm{y}$ e $\mathrm{z}$, respectivamente.

$\mathrm{R}_{\mathrm{x}}, \mathrm{R}_{\mathrm{y}}$ e $\mathrm{R}_{\mathrm{z}}$ representam quaisquer termos adicionais (fontes ou sorvedouros) nas direções $\mathrm{x}, \mathrm{y}$ e $\mathrm{z}$, respectivamente.

Os termos de perda viscosa, nas direções $\mathrm{x}, \mathrm{y}$ e $\mathrm{z}$, podem ser assim determinados:

$$
\begin{aligned}
& \mathrm{T}_{\mathrm{x}}=\frac{\partial}{\partial \mathrm{x}}\left(\mu_{\mathrm{e}} \frac{\partial \mathrm{v}_{\mathrm{x}}}{\partial \mathrm{x}}\right)+\frac{\partial}{\partial \mathrm{y}}\left(\mu_{\mathrm{e}} \frac{\partial \mathrm{v}_{\mathrm{y}}}{\partial \mathrm{x}}\right)+\frac{\partial}{\partial \mathrm{z}}\left(\mu_{\mathrm{e}} \frac{\partial \mathrm{v}_{\mathrm{z}}}{\partial \mathrm{x}}\right) \\
& \mathrm{T}_{\mathrm{y}}=\frac{\partial}{\partial \mathrm{x}}\left(\mu_{\mathrm{e}} \frac{\partial \mathrm{v}_{\mathrm{x}}}{\partial \mathrm{y}}\right)+\frac{\partial}{\partial \mathrm{y}}\left(\mu_{\mathrm{e}} \frac{\partial \mathrm{v}_{\mathrm{y}}}{\partial \mathrm{y}}\right)+\frac{\partial}{\partial \mathrm{z}}\left(\mu_{\mathrm{e}} \frac{\partial \mathrm{v}_{\mathrm{z}}}{\partial \mathrm{y}}\right) \\
& \mathrm{T}_{\mathrm{z}}=\frac{\partial}{\partial \mathrm{x}}\left(\mu_{\mathrm{e}} \frac{\partial \mathrm{v}_{\mathrm{x}}}{\partial \mathrm{z}}\right)+\frac{\partial}{\partial \mathrm{y}}\left(\mu_{\mathrm{e}} \frac{\partial \mathrm{v}_{\mathrm{y}}}{\partial \mathrm{z}}\right)+\frac{\partial}{\partial \mathrm{z}}\left(\mu_{\mathrm{e}} \frac{\partial \mathrm{v}_{\mathrm{z}}}{\partial \mathrm{z}}\right)
\end{aligned}
$$

Sendo o fluido incompressível e a viscosidade constante, os termos $\mathrm{T}_{\mathrm{x}}, \mathrm{T}_{\mathrm{y}}$ e $\mathrm{T}_{\mathrm{z}}$, nas eqs.(2), (3) e (4), foram desprezados.

\section{Análise por elementos finitos da máquina recolhedora}

Neste trabalho, a simulação da fluidodinâmica na máquina recolhedora foi realizada somente com o fluxo de ar, sem a presença dos frutos de café no sistema de transporte pneumático. Essa simulação, sem a presença dos frutos de café, foi preestabelecida devido ao grau de dificuldade encontrado para analisar o comportamento da massa dos frutos de café no sistema de transporte pneumático da máquina recolhedora.

A modelagem foi feita em estado permanente, admitindo regime do fluxo de ar turbulento. $\mathrm{O}$ método de solução usado na discretização do sistema foi o FLOTRAN, juntamente com o elemento 
tridimensional (FLUID142), proposto e contido na biblioteca do ANSYS 5.7. Foi construído um modelo para a estrutura da máquina, utilizando suas propriedades e condições de simetria.

Além disso, foi analisada a estrutura da máquina recolhedora como um bloco, constituída de três partes: a) duto de entrada dos frutos de café; b) caixa do depósito dos frutos de café, e c) duto de saída do fluxo de ar. A geometria da máquina recolhedora utilizada no modelo é apresentada na Figura 3.

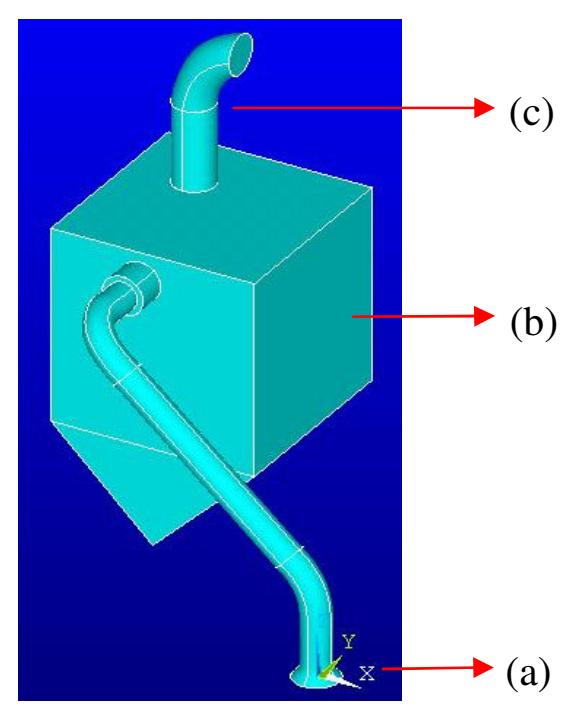

FIGURA 3. Geometria da máquina recolhedora para análise de elemento finito, vista em perspectiva.

Dessa forma, a simulação computacional gerou, por meio do programa ANSYS, a malha de elementos da máquina (Figura 4).

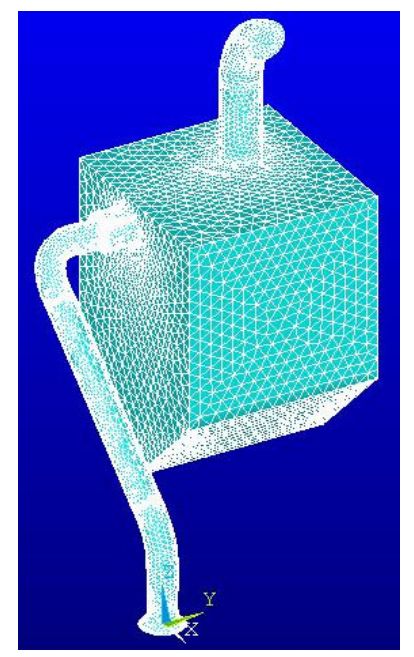

FIGURA 4. Discretização do modelo da máquina recolhedora em elementos finitos.

$\mathrm{Na}$ modelagem dessa máquina recolhedora, foi preestabelecido que o maior refinamento da malha seria no duto de entrada (captador) dos frutos de café até a caixa gravitacional e no duto após a caixa gravitacional até o ventilador, com a finalidade de obter resolução mais precisa.

A máquina recolhedora foi dividida em 170.753 elementos do tipo FLUID142, resultando em um total de 32.415 nós ao final da discretização (Figura 5). 


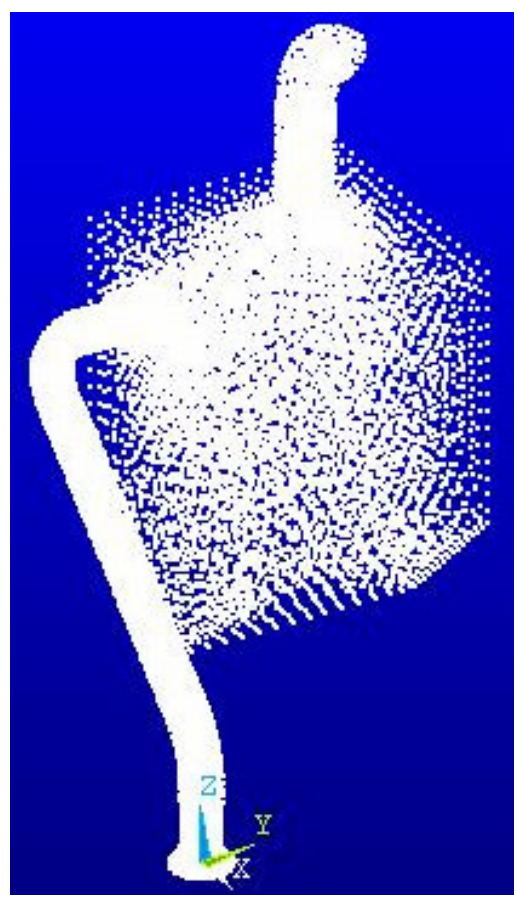

FIGURA 5. Nós da malha de elementos finitos da máquina recolhedora de frutos de café.

As condições de contorno utilizadas foram as seguintes: a) velocidades nulas nas paredes internas da máquina; b) velocidade do ar constante na saída do duto do ventilador, e c) pressão igual a zero na área de entrada de ar.

\section{Determinação experimental da pressão estática na máquina recolhedora}

As pressões estáticas nos dutos da máquina recolhedora foram determinadas com a finalidade de comparar esses valores obtidos experimentalmente com os resultados determinados pelo programa ANSYS. Para determinar a pressão estática nos dutos da máquina recolhedora, foram feitos dez furos nos mesmos. Nesses furos, foram fixados tubos de cobre de comprimento de $50 \mathrm{~mm}$ e diâmetros interno e externo de 7,76 e 9,54 mm, respectivamente, conforme apresentado na Figura 6. Os furos foram feitos a partir do captador até a entrada do fluxo de ar no ventilador. A seguir, a pressão estática foi determinada utilizando manômetro em "U".

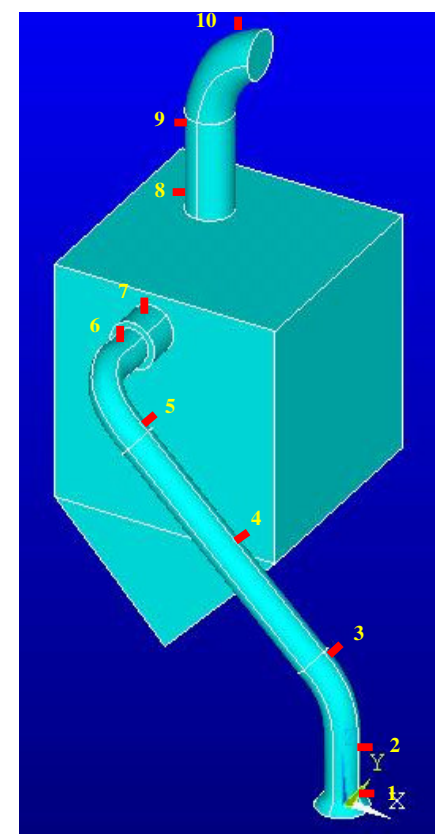

FIGURA 6. Ponto de leitura da pressão estática na máquina recolhedora, vista em perspectiva. 


\section{RESULTADOS E DISCUSSÃO}

$\mathrm{O}$ resultado do perfil do vetor velocidade $\left(\mathrm{m} \mathrm{s}^{-1}\right)$ no captador do sistema de transporte pneumático da máquina recolhedora é apresentado na Figura 7. Observou-se que o perfil do vetor velocidade do sistema de transporte pneumático da máquina recolhedora apresentou, na extremidade do duto captador, marcada por um círculo vermelho, valores que variaram de 0 a $8,8 \mathrm{~m} \mathrm{~s}^{-1}$. Esse intervalo foi determinado pelo programa ANSYS como um ponto crítico, porque ocorre redução do diâmetro de 220 para $145 \mathrm{~mm}$. Esse intervalo foi inferior à menor velocidade terminal calculada dos frutos de café $\left(13,5 \mathrm{~m} \mathrm{~s}^{-1}\right)$, podendo dificultar o recolhimento dos frutos de café no terreiro. Para corrigir esse problema, foi instalada, no fundo do captador, uma chapa de 2,0 $\mathrm{mm}$ de espessura, com a função de direcionar e encaminhar os frutos de café para dentro do duto (Figura 8), onde o perfil do vetor velocidade permanece entre 17,6 a 26,4 $\mathrm{m} \mathrm{s}^{-1}$ (Figura 7).

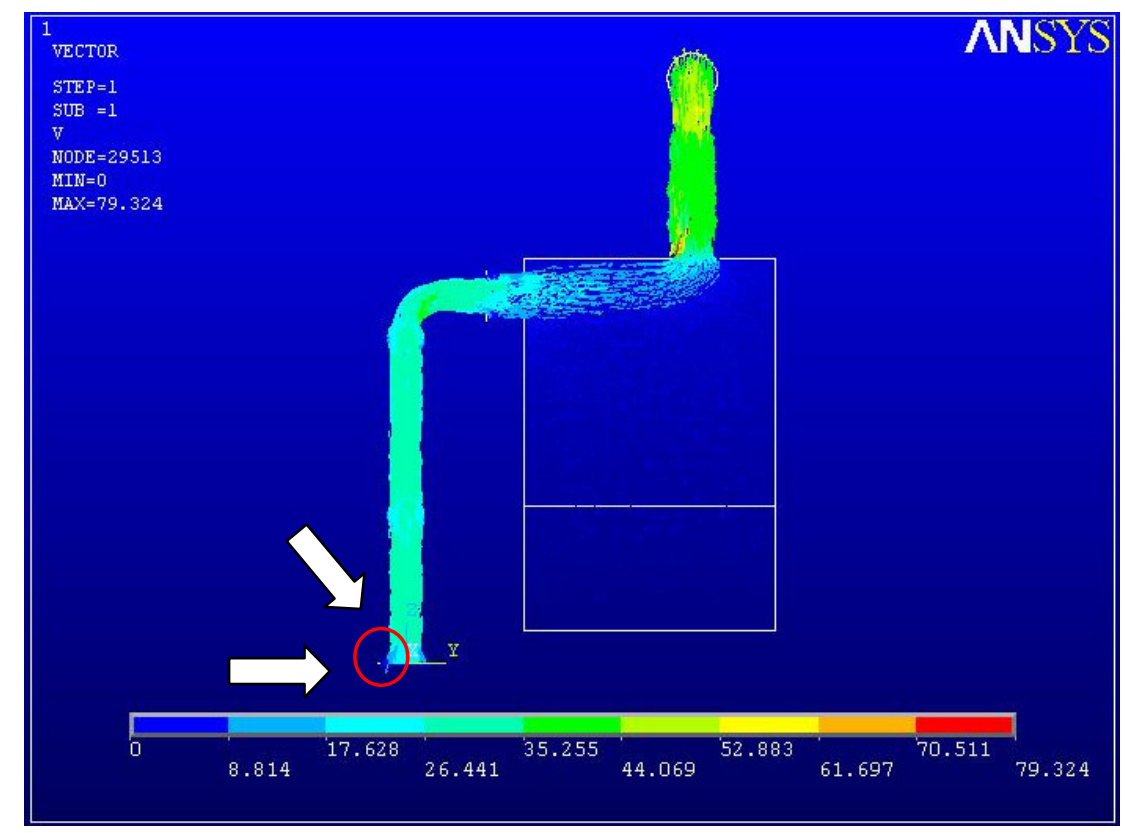

FIGURA 7. Vista frontal do perfil do vetor velocidade no captador do sistema de transporte pneumático da máquina recolhedora.

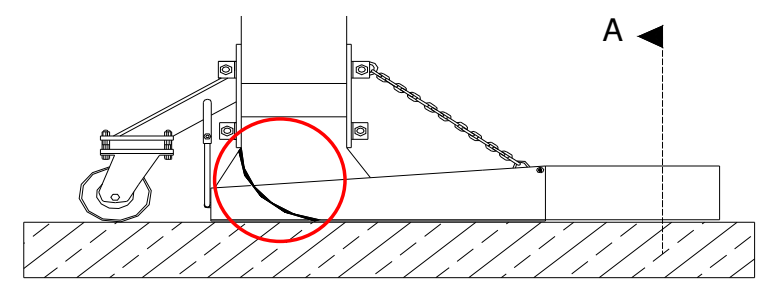

FIGURA 8. Detalhe da chapa colocada no captador da máquina recolhedora.

Na Figura 9, são apresentados os perfis do vetor velocidade dentro dos dutos e da caixa do depósito dos frutos de café. Observa-se, no duto do captador até a caixa do depósito, que o perfil do vetor velocidade permanece em um intervalo de 26,4 a $35,3 \mathrm{~m} \mathrm{~s}^{-1}$. Esse intervalo é superior à velocidade terminal máxima dos frutos de café, que foi de $15,5 \mathrm{~m} \mathrm{~s}^{-1}$, com o teor de água de $41,1 \%$. Segundo ROA et al. (1999), as velocidades mínimas do ar requerido para o transporte pneumático horizontal e vertical do café em coco recém-lavado são de 17,9 e 19,7 $\mathrm{m} \mathrm{s}^{-1}$, respectivamente, em dutos com diâmetros de $160 \mathrm{~mm}$. Para diâmetros de 107,7 mm, as velocidades mínimas indicadas são de 11,9 e 13,2 $\mathrm{m} \mathrm{s}^{-1}$, para o transporte pneumático horizontal e vertical, respectivamente. Portanto, o perfil do vetor velocidade entre 26,4 e $35,3 \mathrm{~m} \mathrm{~s}^{-1}$ aplicado foi suficiente no processo de transporte pneumático dos frutos de café no terreiro. 


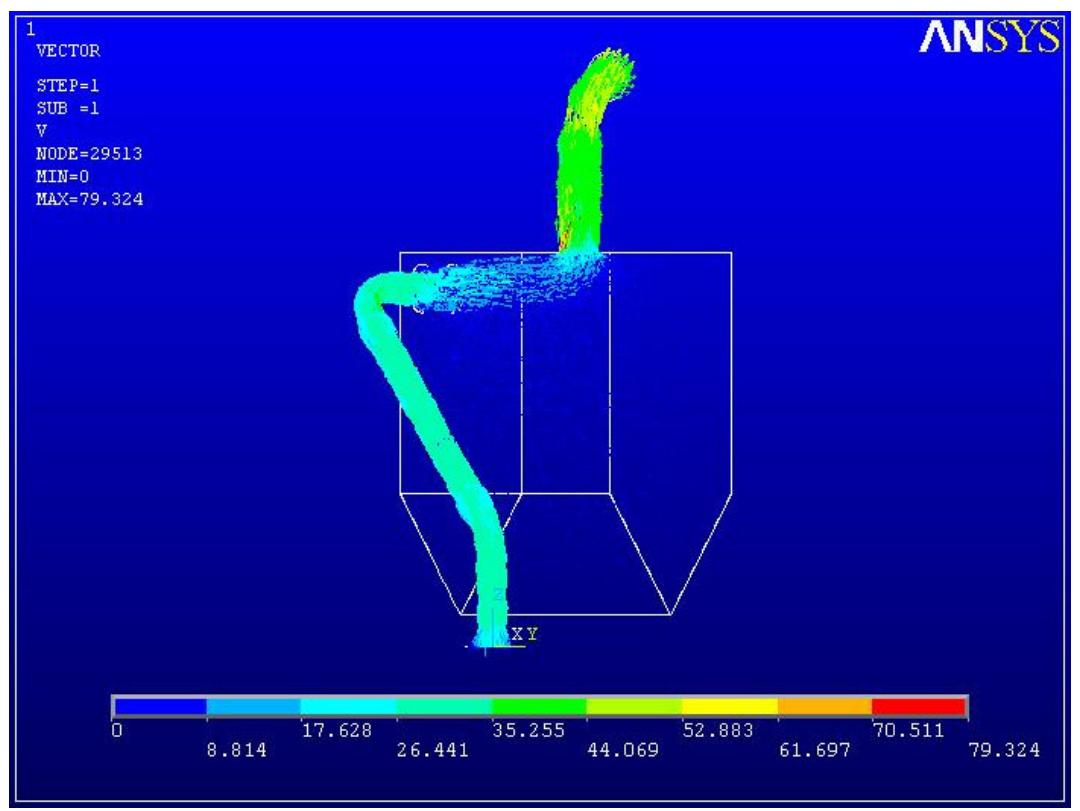

FIGURA 9. Vista lateral do perfil do vetor velocidade nos dutos e na caixa do depósito da máquina recolhedora.

No interior do depósito da máquina recolhedora, houve redução do perfil do vetor velocidade para o intervalo de 8,8 a 17,6 $\mathrm{m} \mathrm{s}^{-1}$, devido à expansão da seção na entrada da caixa do depósito, resultando na desaceleração dos frutos de café e, conseqüentemente, o depósito no fundo da caixa. Observa-se, ainda, que o perfil do vetor velocidade após o depósito variou de 35,3 a 44,1 $\mathrm{m} \mathrm{s}^{-1}$.

$\mathrm{Na}$ Figura 10, é apresentada a pressão (Pa) de sucção na máquina recolhedora. Houve aumento da pressão negativa (ou pressão de sucção) a partir da entrada do duto captador, que foi de zero (condição de contorno inicial), até o valor negativo de $2,2 \mathrm{kPa}$ na saída do fluxo de ar no duto superior. A pressão de sucção variou de 0 a $0,93 \mathrm{kPa}$ negativo, da entrada do duto captador até a caixa do depósito, devido à sucção do transporte pneumático dos frutos de café do terreiro.

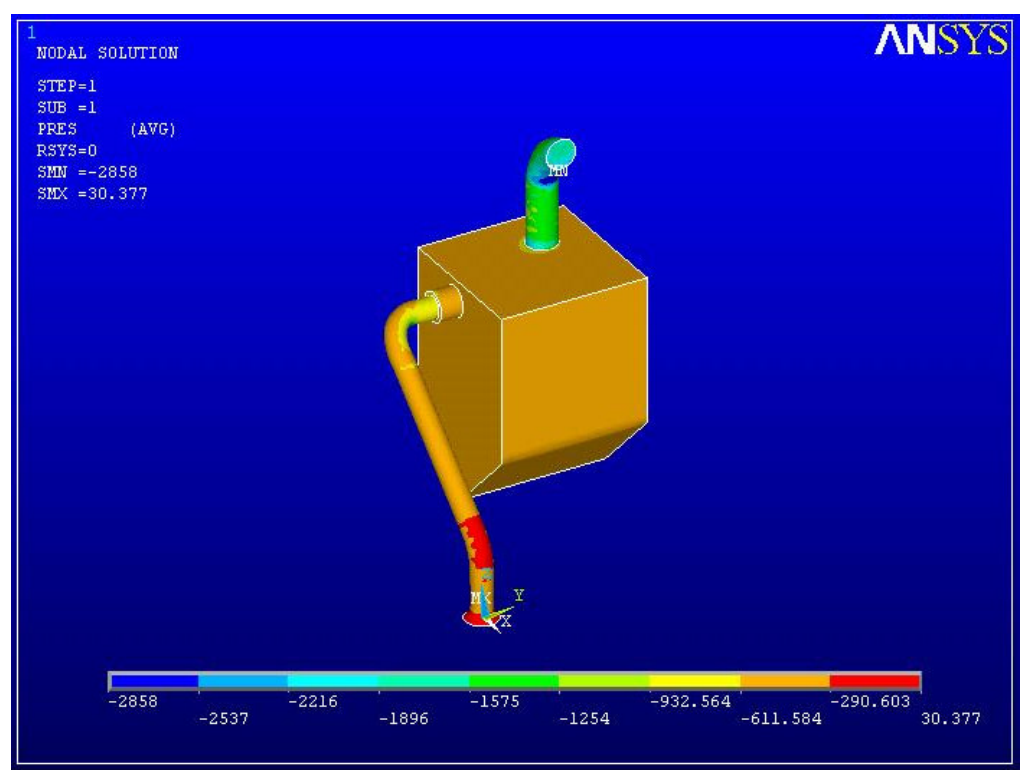

FIGURA 10. Vista geral da pressão de sucção nos dutos e na caixa do depósito da máquina recolhedora.

Na Figura 11, são apresentadas as pressões estáticas experimentais e as simuladas pelo programa ANSYS na máquina recolhedora. 


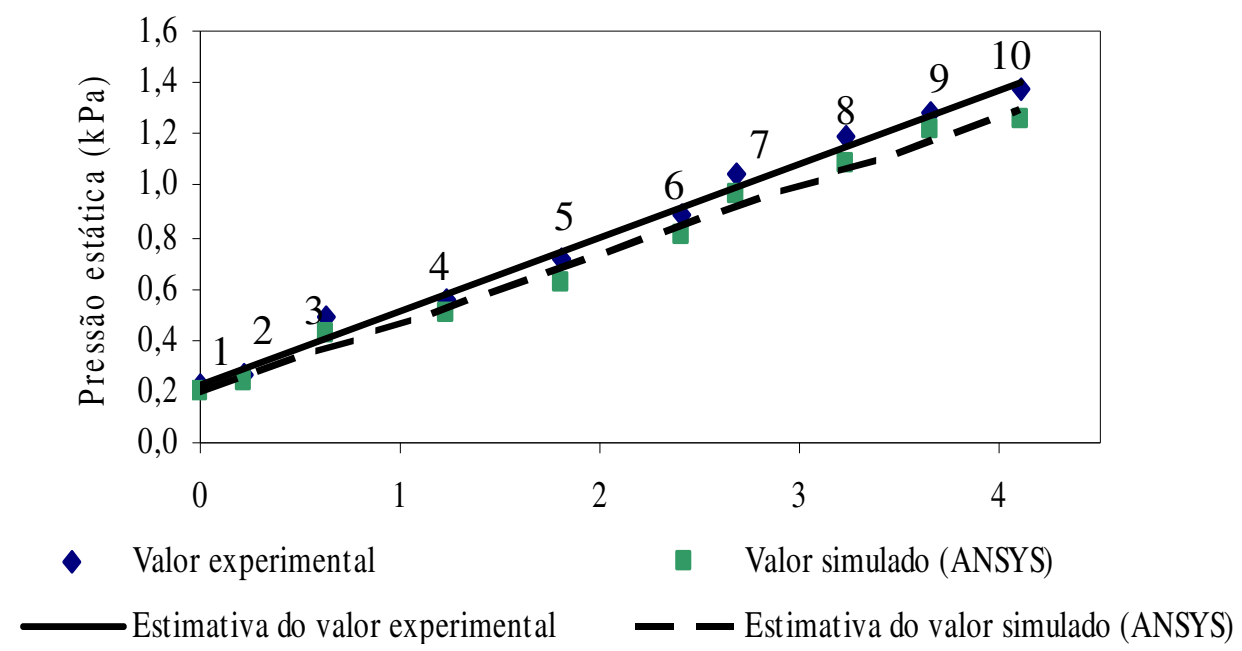

FIGURA 11. Comparação entre pressões estáticas experimentais e as simuladas pelo programa ANSYS na máquina recolhedora.

Na Figura 11, verifica-se que os valores obtidos pelo programa ANSYS foram inferiores àqueles determinados experimentalmente. Isso pode ser devido à condição de contorno (velocidades nulas nas paredes internas da máquina recolhedora) preestabelecida na construção do modelo. $\mathrm{O}$ erro relativo médio envolvido nos valores simulados de pressão, usando a técnica de elementos finitos (ANSYS), foi de 9,2\%, sendo o menor valor de $6 \%$ e o maior de $14 \%$.

\section{CONCLUSÕES}

O programa ANSYS, por meio da modelagem, identificou intervalos que dificultaria o transporte pneumático dos frutos de café nos dutos da máquina recolhedora. Esses problemas foram corrigidos antes da construção da máquina, reduzindo custos e testes experimentais.

Os valores de pressão obtidos, usando a técnica dos elementos finitos, foram próximos aos valores experimentais, validando, assim, a utilização do programa ANSYS para esse tipo de análise.

O refinamento da malha, nos dutos da máquina recolhedora, foi o principal fator que implicou a diminuição do erro relativo entre os valores simulados de pressão e os obtidos experimentalmente.

A modelagem desenvolvida atendeu às necessidades propostas no projeto da máquina para o recolhimento dos frutos de café em terreiro, utilizando transporte pneumático.

\section{REFERÊNCIAS}

ANSYS 5.7. Fluid flow fundamental. In: KOHNKE, P. (Ed.) Ansy theory reference manual. Houston: ANSYS, 2001.

GARCIA, R.F. Otimização do projeto de componentes de uma colhedora de forragem. $1998.78 \mathrm{f}$. Dissertação (Mestrado em Máquinas e Mecanização Agrícola) - Universidade Federal de Viçosa, Viçosa - MG, 1998.

HAGHIGHI, K.; SMITH, M.G.; KRUTZ, G.W. On board testing and shape optimization of engine mount bracket. Applied Engineering in Agriculture, St. Joseph, v.6, n.4, p.382-9, 1990.

HUEBNER, K.H.; THORNTON, E.A.; BYROM, T.G. The finite element method for engineers. New York: John Wiley, 1995. 627 p.

KNIGHT, C.E. The finite element method in mechanical design. Boston: PWS-KENT, 1993. 326 p. 
MAGALHÃES, A.C. Desenvolvimento e avaliação de uma máquina recolhedora de café em terreiro utilizando transporte pneumático. 2003. 110 f. Tese (Doutorado em Máquinas e Mecanização Agrícola) - Universidade Federal de Viçosa, Viçosa - MG, 2003.

NORTON, R.L. Design of machinery: an introduction to the synthesis and analysis of mechanisms and machines. New York: McGraw-Hill, 1992. 714 p.

REDDY, J.N. An introduction to the finite element method. New York: McGraw-Hill, 1993. 684 p. ROA, M.G.; OLIVEROS, T.C.E.; ÁLVAREZ, G.J.; RAMÍREZ G.C.A.; SANZ U.J.R.; DÁVILA, A. M.T.; ÁLVAREZ, H.J.R.; ZAMBRANO, F.D.A.; PUERTA, Q.G.I.; RODRÍGUEZ, V.N. Beneficio ecológico del café. Chinchina: CENICAFÉ, 1999. 300 p.

SEGERLIND, L. J. Applied finite element analysis. New York: John Wiley, 1984. 427 p.

SILVA, J.S. Pré-processamento de produtos agrícolas. In: LUCENA, E.M.P; SILVA, J.S. Estrutura, composição e propriedades das sementes. Juiz de Fora: Instituto Maria, 1995. p.23-32.

SRIVASTAVA, K.A.; GOERING, E.C.; ROHRBACH, P.R. Engineering principles of agricultural machines. St. Joseph: ASAE, 1996. 601 p.

ZHU, J.; HAGHIGHI, K.; KRUTZ, G.W.; SMITH, M.G. Harmonic and modal analysis of a diesel engine chassis mount bracket - a finite element approach. Applied Engineering in Agriculture, St. Joseph, v.5, n.4. p.467-74, 1989. 